\title{
非线性科学专辑·评述
}

\section{p53 信号网络的非线性动力学研究}

\author{
张小鹏 ${ }^{(1) 2}$ ，刘锋 ${ }^{(1 *}$ ，王炜 ${ }^{(1 *}$ \\ (1) 南京大学物理学院, 固体微结构国家重点实验室, 南京 210093; \\ (2) 南京大学匡亚明学院, 南京 210093 \\ *联系人, 刘锋, E-mail: fliu@nju.edu.cn; 王炜, E-mail: wangwei@nju.edu.cn
}

收稿日期: 2014-04-23; 接受日期: 2014-07-01

国家重点基础研究发展计划 (编号: 2013CB834104) 和国家自然科学基金 (批准号: 11175084, 11204126, 31361163003) 资助项目

\begin{abstract}
摘要 $\mathrm{p} 53$ 蛋白作为细胞内重要的肿瘤抑制因子, 通过复杂的信号转导网络调节细胞生理活动, 维护基因组 的稳定性与完整性. p53 网络能对细胞遭遇的一系列应激信号 (如 DNA 损伤、致癌基因激活和端粒受损等) 做 出响应, 启动 DNA 修复、细胞周期阻滞、细胞衰老或调亡等来避免基因组缺陷的复制和遗传.p53 蛋白浓度在 细胞应激响应过程中呈现脉冲、开关等动力学行为, 具有丰富的非线性特征. 本文旨在综述 p53 网络动力学 领域的最新进展, 揭示细胞 DNA 损伤响应过程中 p53 动力学与功能之间的潜在联系.
\end{abstract}

关键词非线性动力学, p53 脉冲, 细胞凋亡, 细胞周期阻断, 细胞命运抉择

PACS: 87.16.Yc, 05.45.-a, 87.16.Xa

doi: 10.1360/SSPMA2014-00121

\section{1 引言}

非线性因素存在于众多的动力系统中, 传统的 线性化方法已不能满足求解非线性问题的需要. 自 20 世纪 60 年代以来, 非线性动力学已发展成一门重 要的前沿学科, 主要研究分岔、混沌和孤立子等 ${ }^{[1]}$. 非线性科学的概念与方法被广泛应用于不同研究领 域. 非线性系统的一个典型范例是生命系统. 细胞作 为生物体的基本结构单元, 含有大量由基因和蛋白 质等生物分子构成的信号转导网络; 它们执行着生 物功能, 表现出丰富的动力学行为 ${ }^{[2]}$.

p53 蛋白是细胞内最重要的肿瘤抑制因子之一, $50 \%$ 以上的人类肿瘤与 $\mathrm{p} 53$ 基因突变有关. 在正常生 理条件下, p53 受到其负调控因子 Mdm2 的抑制而处 于未激活状态 ${ }^{[3]}$. 当细胞遭遇应激信号 (如 DNA 损
伤、原癌基因活化和端粒功能受损等), p53 蛋白大量 积累并被激活 [4]. 具有活性的 p53 主要作为转录因 子调控一系列靶基因的表达, 其表达产物调控或参与 DNA 损伤修复、细胞周期阻断、细胞衰老和调亡等 过程 ${ }^{[5]}$. p53 也能通过非转录依赖的机制调控 DNA 损伤修复和细胞调亡等 ${ }^{[6]} . \mathrm{p} 53$ 蛋白与其上下游通路 一起构成了复杂的信号转导网络, 将特定的应激信 号转化为特定的细胞命运 ${ }^{[7]}$. p53 信号网络包含众多 的反馈和前馈等结构, 其信息传递和处理过程蒀含 着丰富的非线性特性 ${ }^{[8]}$.

30 多年来, p53 研究领域取得了大量重要的进 展, 促进了人们对 $\mathrm{p} 53$ 抑癌功能的理解. 研究 p53 网 络的动力学与功能是其中的一个重要研究方向. 2000 年, 以色列魏茨曼科学研究所的 Oren 及其合作者发 现乳腺癌 MCF-7 细胞在受到电离辐射后, p53 蛋白

引用格式: 张小鹏, 刘锋, 王炜. p53 信号网络的非线性动力学研究. 中国科学: 物理学 力学 天文学, 2014, 44: 1311-1318

Zhang X P, Liu F, Wang W. Nonlinear dynamics in the p53 signaling network (in Chinese). Sci Sin-Phys Mech Astron, 2014, 44: 1311-1318, doi: 10.1360/SSPMA2014-00121 


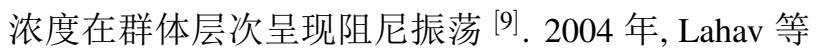
人进一步发现 p53 浓度在单细胞层次呈现数字式的 脉冲, 即脉冲数目随着辐射剂量的增加而增加, 但周 期和幅度几乎不随辐射剂量而变化 ${ }^{[10]}$. 这种数字式 的 p53 响应模式, 与以往的分级响应模式截然不同. 在经典模型中, 一般认为应激信号强度直接调控 p53 蛋白的水平, 进而决定细胞的生死 ${ }^{[11]}$. p53 脉冲的产 生机制及其如何决定细胞命运引起了人们广泛的兴 趣. 在 p53 网络动力学的建模研究中, 非线性动力学 的分岔理论获得了广泛应用.

本文将回顾有关细胞 DNA 损伤响应过程中的 p53 动力学的重要研究进展. 我们探讨了特定 p53 动 力学行为的产生机制, 特别是网络中的正负反馈结 构如何影响 p53 动力学. 我们介绍了理论建模中如 何刻画 p53 的上下游信号因子、多种 p53 翻译后修 饰、辅助因子等对 p53 动力学与功能的调控, 揭示 细胞 DNA 损伤响应过程的分子机制. 我们还讨论了 p53 动力学模式与细胞命运之间的联系, 及其对癌症 治疗所具有的潜在重要性.

\section{$2 p 53$ 信号转导网络}

p53 信号转导网络在抑制肿瘤发生发展方面发 挥着关键的作用. 一般而言, 该网络主要由输入信 号、信号传感器、调控模块、效应器和输出信号等 组成 ${ }^{[12]}$. 输入信号是指一系列可以激活 p53 蛋白的 应激信号, 包括氧化应激、营养贵乏、DNA 损伤、致 癌基因激活、核糖体功能失调和端粒受损等. 在下文 中, 我们主要聚焦于 p53 网络对 DNA 损伤的响应. 如 图 1 所示, 多种途径 (如电离辐射、紫外辐射及抗癌 药物等) 可导致 DNA 损伤. DNA 损伤能活化多种磷 酸化激酶 (如 ATM, ATR, DNA-PK, CHK1, CHK2 等), 它们充当了信号传感器. 这些激酶会磷酸化 p53 等 蛋白, 解除 Mdm2 对 p53 的抑制, 促进 p53 在细胞内 的积累. 激活的 p53 可作为转录因子调节大量靶基 因的表达. 实验发现, p53 活化呈现渐进方式. 在损伤 响应早期, Ser-15 和 Ser-20 等位点的磷酸化可部分激 活 p53, 导致 Wip1 和 p21 等促存活基因的表达; 而严 重的损伤可进一步引起 Ser-46 位点的磷酸化, 导致 p53 的完全激活, 进而表达 PTEN 和 p53AIP1 等促调
亡基因 ${ }^{[13-15]}$. p53 靶基因的表达产物 p53DINP1 可作 为辅助因子协助 HIPK2 激酶磷酸化 p53 的 Ser-46 位 点 ${ }^{[16]}$. 部分靶基因的表达产物可以进一步调节 p53 活性, 组成了多个反馈回路, 包括 p53-Mdm2, p53Wip1-ATM 负反馈回路和 p53-PTEN-PIP3-Mdm2 正反 馈回路等. 这些反馈回路对调节 p53 动力学和功能起 着重要的作用. 某些 p53 靶基因表达物则直接参与了 对细胞生理活动的调控, 充当了效应器的角色. 在可 修复细胞中, p21 引起细胞周期阻断; 在严重受损的

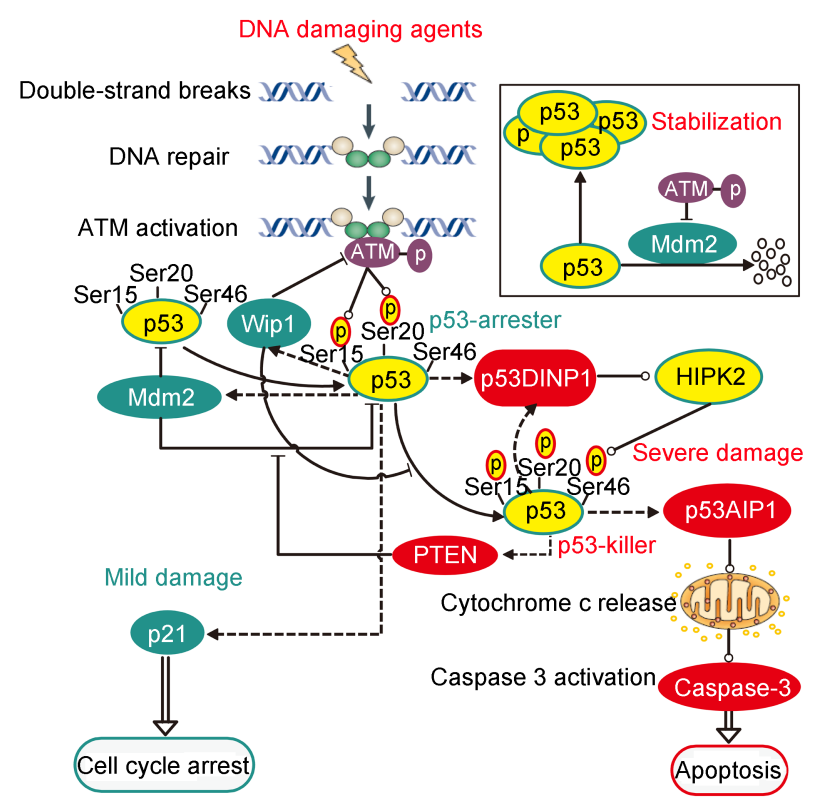

图 1 (网络版彩图)p53 信号网络决定细胞命运的工作机理示 意图

多种因素可导致 DNA 损伤, 细胞内存在损伤修复机制. DNA 损伤导致 ATM 激酶的自磷酸化活化; ATM 再通过磷酸化底物 使 p53 稳定并活化 (见插图). 根据其磷酸化状态的不同, 活化 的 p53 被分为 p53-arrester 和 p53-killer, 前者可表达 p21 引起细 胞周期阻断, 后者则通过表达 p53AIP1 等引起细胞调亡. 图中 用虚线表示 $\mathrm{p} 53$ 转录激活靶基因, 用实线表示其他过程. 末端 圆圈表示促进, 末端短线表示抑制, 双线箭头表示引起不同的 细胞命运

Figure 1 (Color online) Schematic diagram of cell-fate decision by the p53 signaling network. Multiple agents can induce DNA damage, and DNA repair proteins are recruited to fix the damage. DNA damage leads to activation of the ATM kinase via autophosphorylation. ATM phosphorylates its substrates; consequently, p53 is stabilized and activated (see the inset). Based on its phosphorylation status, active p53 is divided into p53-arrester and p53-killer; the former induces p21 to arrest the cell cycle, whereas the latter induces pro-apoptotic genes such as p53AIP1 to trigger apoptosis. Dashed lines denote gene expression mediated by p53, while solid lines denote other processes. Circle- and bar-headed lines denote the promotion and inhibition, respectively, of state transitions or protein interactions. 
细胞中, p53AIP1 等促进细胞色素 $\mathrm{c}$ 从线粒体释放到 细胞质, 进而激活 caspase- 3 等调亡执行蛋白, 引起细 胞调亡. 因此, 通过复杂的信号转导, p53 网络将各种 应激信号转化为特定的细胞命运, 从而维护细胞基 因组的稳定性与完整性, 遏制肿瘤的发生发展.

\section{3 p53 脉冲的产生机制与可激发性}

人们最初是在乳腺癌 MCF-7 细胞系中观察到 p53 振荡动力学的. 细胞受电离辐射后, p53 浓度 在群体层次呈现阻尼振荡 [9], 但在单细胞层次则表 现为数字式脉冲, 脉冲的数目与损伤强度存在正关

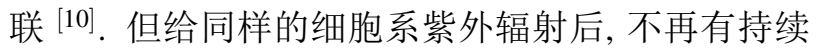
的 $\mathrm{p} 53$ 脉冲, 只有单一的宽幅 p53 脉冲, 其幅度与损 伤强度相关 ${ }^{[17]}$. 电离辐射和某些癌症化疗药物可诱 导 DNA 双链断裂, 而紫外辐射则主要诱导 DNA 单 链断裂, 不同的 DNA 损伤类型导致了不同的 $\mathrm{p} 53$ 动 力学. 除了 MCF-7 细胞, 人们也在其他几个肿瘤细胞 系中发现了 DNA 双链断裂诱导的 p53 脉冲 [18]. 因 此, p53 的动力学模式具有细胞类型和应激信号种类 相关的特异性.

继 p53 振荡被发现后, 一系列理论工作围绕其产 生机制展开. 2000 年, 研究人员在实验发现 p53 阻尼 振荡的同时, 也通过理论建模提出, 含有时间延迟的 p53-Mdm2 负反馈导致了 p53 振荡的发生 ${ }^{[9]} .2005$ 年, 美国 IBM 沃森研究中心的 Ma 及其合作者进一步从 理论上探究了 p53 脉冲的产生机制 [19]. 他们构建了 包含 DNA 损伤产生和修复模块、ATM 活化模块和 p53-Mdm2 振荡模块的整合模型, 并在第一个模块中 引入了随机性. 数值模拟结果很好地重复了 $\mathrm{p} 53$ 数字 式脉冲的实验结果, 表明群体层次的阻尼振荡是具有 差异性的单细胞 $\mathrm{p} 53$ 脉冲动力学平均的结果. 除了强 调 p53-Mmd2 负反馈对于 $\mathrm{p} 53$ 脉冲的重要性, 他们还 提出, ATM 作为损伤传感器所呈现的类开关式动力 学是维持 p53 脉冲形状均一的重要保证. 2008 年, 哈 佛大学的 Lahav 小组采用实验观测和理论建模相结 合的方法, 发现单一的 p53-Mdm2 负反馈并不足以使 p53 脉冲持续, 而 p53-Wip1-ATM 负反馈对于维持形 状均一的 p53 脉冲则是必需的 [20]. 他们提出, p53 脉 冲是由上游的 ATM 脉冲驱动的, 即 DNA 损伤导致
的 ATM 反复激活导致了 $\mathrm{p} 53$ 脉冲的发生.

另一方面, 美国弗吉尼亚理工大学的 Tyson 研究 组在他们的理论研究中强调了耦合的正负反馈结构

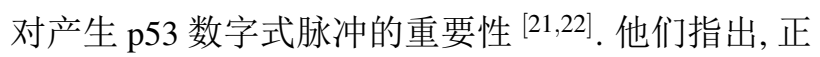
反馈结构有助于系统产生亚临界霍普夫分岔, 从而产 生大幅度的 p53 振荡. 该研究提出了 p53 网络产生数 字式脉冲的另一种机制 [21]. 在后续的研究中, 该研究 组又指出可能存在 4 种耦合的正负反馈结构来诱发 DNA 损伤响应中的 p53 脉冲 [22]. 负反馈结构对产生 p53 脉冲的重要性已在 MCF-7 细胞中为实验所证实, 但正反馈结构对 $\mathrm{p} 53$ 脉冲的重要性还有待实验验证. 在其他细胞系中, 正反馈可能在 p53 脉冲的产生中扮 演重要的角色.

Lahav 等人还发现在无应激的正常分裂的细胞 中有自发产生的 p53 脉冲存在; 这种脉冲是由 DNA 复制过程中产生的瞬时损伤所引起的 ${ }^{[23]}$. 而且, 应激 诱导和自发产生的 p 53 脉冲均具有可兴奋性, 被看作 是 p53 脉冲区别于普通振荡的一个重要特性. p53 脉 冲的可激发性是指一个短暂的 ATM 脉冲 (持续时间 为 1 小时或更短) 可驱动一个完整的 $\mathrm{p} 53$ 脉冲 (脉 冲宽度为 4-7 小时). 有研究表明, DNA 损伤响应中 ATM 依赖的 Mdm2 快速降解可能是 p53 脉冲具有可 激发性的一个必要条件 [17]. 目前, 尚不清楚这种可 兴奋性在 p53 行使生物功能的过程中扮演何种角色, 值得进一步研究. 南京大学的沈萍萍研究组对非应 激条件下 $\mathrm{p} 53$ 脉冲的自发产生过程进行了建模研究, 确认可兴奋性是自发产生脉冲的重要条件 [24].

\section{4 p53 网络决定细胞命运的动力学机制}

\section{1 p53靶基因的选择性表达决定细胞生死}

关于 $\mathrm{p} 53$ 脉冲, 一个更深入的问题是它与 $\mathrm{p} 53$ 生 物功能之间的关系. p53 主要作为转录因子, 调控一 系列与细胞周期阻断和调亡相关的靶基因, 决定细胞 的生死, 抑制肿瘤的发生发展 ${ }^{[5]}$. 人们曾认为 $\mathrm{p} 53$ 蛋 白对 DNA 损伤呈现一种分级响应模式: 轻度损伤激 发少量 p53 蛋白, 介导促细胞周期阻断基因 (如 p21) 的表达, 细胞经历短暂的细胞周期阻断后, 恢复到正 常增殖状态; 当损伤较严重时, 大量的 p53 蛋白聚 
积, 表达促调亡基因 (如 PUMA 等), 细胞最终走向死 亡 [11]. 因此, 细胞命运是由依赖于损伤强度的 p53 蛋白水平决定的. 显然, 当存在 p53 脉冲时, 经典的 分级响应模式已不再适用于描述细胞命运抉择过程. 近年来, p53 脉冲调控细胞命运抉择的动力学机制已 成为 $\mathrm{p} 53$ 领域的研究热点.

2007 年, Tyson 研究组构建了 p53 网络对 DNA 损 伤响应的理论模型, 探讨 $\mathrm{p} 53$ 脉冲决定细胞命运的动 力学机制 ${ }^{[22]}$. 该模型中 $\mathrm{p} 53$ 脉冲是由耦合的正负反 馈结构产生的. 根据不同的磷酸化状态, 激活的 p53 被分为三种形式. 基于数值模拟结果, 他们提出假说: p53 脉冲的数目决定细胞命运. 对于轻度损伤, 少量 p53 脉冲引起细胞周期阻断, 细胞可在修复完成后回 归正常状态. 对于严重损伤, 持续产生的 p 53 脉冲激 活下游调亡程序杀死不可修复细胞. 该研究对 $\mathrm{p} 53$ 脉 冲的生物功能做了有意义的探索.

2009 年, 我们构建了更为全面的应答 DNA 损伤 的 p53 网络模型 ${ }^{[25]}$. 该模型整合了 DNA 损伤产生 和修复、ATM 损伤感知、p53-Mdm2 振荡发生器和 细胞命运抉择等四个模块, 可全面刻画 p53 网络对 DNA 损伤做出响应的动力学行为. 特别地, 模型中假 设 p53 对损伤修复过程有双重调控效应, 即 p53 可促 进轻度损伤的修复, 而抑制严重损伤的修复; 该假设 调和了以往看似矛盾的实验结果. 随机性在第一个 模块中被引入, 经过网络传递使得最终的细胞命运 呈现多样性. 我们借鉴 Tyson 等人根据 p53 磷酸化状 态对其进行分类的做法, 将激活的 p53 分为两种形 式: p53-arrester(初步磷酸化的 p53, 可转录细胞周期 阻断相关的基因) 和 p53-killer(进一步磷酸化的 p53, 可转录促凋亡相关的基因). 该模型中, p53 脉冲是由 耦合的正负反馈回路在 DNA 损伤驱动下产生的. 研 究表明, 对于轻度损伤, 仅有 p53-arrester 形式的脉冲 被激发, 细胞经历暂态的生长停滞后回到正常状态; 对于严重损伤, 在一定数目的 p53-arrester 脉冲之后, p53-killer 被激活, 并诱导细胞调亡. 模型提出的 p53 对损伤修复的双重调控机制, 可最大程度地减少细胞 命运抉择过程中的个体差异性, 使得低辐射剂量下大 部分细胞得以存活, 而高辐射剂量下大部分细胞都被 杀死, 这对癌症的放疗具有重要意义. 相比 p53 蛋白 浓度, 由 p53 脉冲数目决定细胞命运更具有鲁棒性和
灵活性, 可避免随机因素引起的细胞非正常死亡. 该 模型中, 细胞凋亡是由 p53-killer 脉冲来驱动的; 细胞 做出凋亡的抉择后, 往往还需一段时间才能被杀死, 因而该机制在调亡执行的效率上有待提高.

在后续工作中, 我们针对未发生严重基因突变 的 MCF-10A 细胞系, 构建了一个新的 p53 网络对 DNA 损伤响应的整合模型. 该模型可实现一种更优 化的 $\mathrm{p} 53$ 响应模式, 即 $\mathrm{p} 53$ 脉冲和持续高水平激活 分别引起细胞周期阻断和细胞调亡 ${ }^{26]}$. 该模型主要 考虑了三个 $\mathrm{p} 53$ 相关的反馈回路: p53-Mdm2 负反 馈、p53-Wip1-ATM 负反馈和 p53-PTEN-Mdm2 正反 馈, 它们能精细调控 $\mathrm{p} 53$ 的动力学与功能. 激活的 $\mathrm{p} 53$ 仍然被分为 p53-arrester 和 p53-killer. p53-arrester 主要 表达 p21 和 Wip1, p53-killer 则表达 PTEN 和 p53AIP1 等. 研究发现, 轻度损伤的细胞中 p 53 呈现少数几个 脉冲; 严重损伤的细胞中 p53 动力学表现出两阶段 性. 在第一阶段, 两个负反馈占主导, p53 蛋白呈现一 系列 p53-arrester 的脉冲. 在第二阶段, p53 转变为具 有促凋亡活性的 killer 形式, PTEN 被转录激活, p53PTEN-Mdm2 正反馈开始占主导, p53 水平进一步升 到一个较高的稳态. 高水平的 $\mathrm{p} 53$ 促使细胞快速调 亡. 因此, p53 水平和活性在细胞响应过程中通过渐 进的方式被调控: 初步激活的低幅度脉冲引起细胞 周期阻断, 而完全激活的高水平 p53 则引起细胞凋 亡. p53 网络结构的时序性调控导致了 p53 动力学 的两阶段性转变: 负反馈主导下呈现 p53 脉冲, 正反 馈占主导则呈现开关动力学, p53-arrester 到 p53-killer 的转变则是上述变化的内在原因. 总之, 该研究提出 了一种全新的 p 53 动力学响应模式, 使得 p53 响应兼 具灵活性与鲁棒性, 可被看作是某种优化的应激响 应方式.

p53 蛋白 Ser-46 位点的磷酸化对其表达促调亡 的靶基因至关重要; HIPK2 是调节该磷酸化过程的一 种激酶 ${ }^{[27]}$. 有趣的是, HIPK2 的降解受到 Mdm2 的调 控 ${ }^{[28]}$, 而 HIPK2 引起的 p53 磷酸化会导致 Mdm2 的 表达下调 [29]. 我们建模探讨了 Mdm2 和 HIPK2 的 相互调控对 p53 活性的影响 [30]. 模型详细考虑了下 调 Mdm2 表达的三种方式: 下调转录、促进降解和 通过 PTEN 抑制 Mdm2 入核. 模拟结果表明, Mdm2 对 HIPK2 的降解有利于可修复细胞的存活, 而 Mdm2 
表达的下调对于诱导细胞调亡则是必须的. 3 种下 调 Mdm2 的机制在调亡过程中可相互补偿, 确保通 过 p53 诱导的调亡来杀死严重损伤的细胞.

p53 靶向的 microRNA, 如 miR-605 和 miR-34a 对 p53 活性有显著的调控作用. miR-605 可抑制 Mdm2 的翻译, 促进 $\mathrm{p} 53$ 的激活 ${ }^{[31]}$; miR-34a 主要抑制某些 抗调亡蛋白的合成, 在 p53 信号通路的下游起促调亡 作用 [32]. 通过理论建模, 我们刻画了 miR-605 和 miR$34 \mathrm{a}$ 在 p53 网络中扮演的不同角色 ${ }^{[33]}$. 在 DNA 严重 受损的细胞中, $\mathrm{p} 53$ 动力学表现出两阶段性: 第一阶 段的脉冲和第二阶段的持续激活. miR-605 通过下调 $\mathrm{Mdm} 2$ 的表达来增加 $\mathrm{p} 53$ 脉冲的幅度, 进而加快 $\mathrm{p} 53$ 动力学由脉冲转变为高水平稳态的进程; miR-34a 直 接下调抗调亡基因 Bcl-2 的表达, 促进促调亡蛋白如 p53AIP1 的激活. 我们提出, miR-605 与 miR-34a 的协 同作用提高了调亡发生的鲁棒性.

p53 辅助因子对 p53 靶基因的选择性表达和细 胞命运抉择也有重要的影响. 研究发现, p53 靶基因 表达产物 Hzf 同时也充当其辅助因子, 特异性地促 进 p53 对 p21 的表达, 促进细胞存活; 但在严重损伤 的细胞中, Hzf 会被降解掉, Bax 得以表达而诱发细 胞调亡 [34]. p53 的另一个辅助因子 ASPP1/2(在后文 中统称为 ASPP) 则会特异性地促进 p53 表达促凋亡 基因, 如 PUMA 和 Bax 等 [35]. 我们同时考虑 Hzf 和 ASPP, 研究性质不同的辅助因子如何协同调控细胞 命运抉择 [36]. 结果表明, 在轻度受损的细胞中, Hzf 表达并促进 $\mathrm{p} 21$ 的表达, 从而抑制 E2F1 活化, 引起 暂态的细胞周期阻断, 细胞最终得以存活. 在严重受 损的细胞中, Hzf 在响应后期被降解, p53 对 p21 的表 达显著降低, E2F1 被激活并表达 ASPP. 在 ASPP 辅 助下, p53 激活 Bax 的表达, 引发细胞凋亡. 我们还提 出了两种可能的 Hzf 降解机制. 该研究有助于我们 理解 p53 辅助因子在细胞命运抉择过程中所扮演的 角色.

此外, p53 通路和其他信号通路, 如 E2F1 通路 之间存在着复杂的相互作用, 也会影响 p53 靶基因 表达的选择性 ${ }^{[37]}$. 我们构建了较为复杂的网络模型, 用来揭示 DNA 损伤响应过程中 p53 和 E2F1 如何共 同决定细胞命运 ${ }^{[38]}$. 一方面, p53 可通过表达 p21 抑 制 E2F1 的激活, 引起细胞周期阻断; 另一方面, p53
与 $\mathrm{E} 2 \mathrm{~F} 1$ 协作引起细胞调亡. E2F1 的多个靶基因表 达物参与了 p53 调控的细胞调亡. 比如, E2F1 介导表 达 p53DINP1, 促进 p53Ser-46 的磷酸化, 促使 p53 特 异性表达某些促调亡基因; E2F1 上调 ASPP 的表达, 促进 PUMA 和 Bax 等促凋亡基因的表达. 此外, E2F1 介导 Apaf-1 的表达, 有助于调亡复合体的形成; E2F1 还通过上调 procaspase-3 和 9 等酶原的表达来促进 细胞调亡. 总之, $\mathrm{p} 53$ 和 $\mathrm{E} 2 \mathrm{~F} 1$ 不同的激活状态决定了 细胞的命运: 只有 $\mathrm{E} 2 \mathrm{~F} 1$ 激活时细胞正常增殖; 只有 p53 被激活时细胞周期被阻断; 二者共同激活时细胞 调亡.

\section{$4.2 \mathrm{p53}$ 非转录依赖活性对细胞命运的调控}

p53 除了在细胞核内作为转录因子调控细胞的 应激响应外, 还可以直接移位到线粒体外膜附近, 通 过蛋白 - 蛋白作用直接激活 Bax 等促调亡蛋白, 诱导 细胞凋亡 [6]. 实验发现, 在遭受紫外辐射的 HCT116 细胞内, p53 转录依赖的通路和非转录依赖的通路是 紧密耦合的 ${ }^{[39}$. 我们探讨了细胞核 $\mathrm{p} 53$ 和细胞质 $\mathrm{p} 53$ 在 DNA 损伤响应过程中协同调控细胞调亡的动力 学机制 [40]. 该模型考虑了 p53-Mdm2 负反馈和 p53PTEN-Mdm2 正反馈对 p53 动力学的调控. 研究表明, 只有当损伤较严重时, PTEN 的表达导致 p53 的大量 积累, 细胞核 p53 转录激活 PUMA 和 Bax. PUMA 在 细胞质中结合 Bcl-xL 等抗调亡蛋白, 释放出原先被 Bcl-xL 等结合的 $\mathrm{p} 53$, 最终激活 Bax 导致细胞调亡. 我们发现, p53 在细胞核和细胞质的浓度相对平衡对 高效的细胞调亡是必须的.

在对放射线敏感的器官 (如胸腺与脾脏) 中, 线 粒体 p53 依赖的细胞调亡可以在细胞遭受电离辐射 后 1-2 小时内被快速诱导, 而转录依赖的细胞调亡则 在辐射后 8 小时才开始出现 ${ }^{[41]}$. 基于相关实验结果, 我们构建了 p53 网络响应 DNA 损伤的整合模型. 该 模型包括了细胞核和线粒体 p53 通路, 着眼于刻画它 们在损伤响应过程中的相互作用 [42]. 结果显示, 对射 线敏感的细胞在命运抉择过程中存在两个细胞调亡 的检查点. 损伤特别严重的细胞通过非转录依赖的 p53 途径在反应早期的第一个检查点激活调亡程序. 那些遭受中等强度损伤的细胞则会尝试修复损伤, 以 
转录依赖的 p53 通路发挥主要作用. 一段时间后, 若 损伤修复完成, 细胞得以存活. 若损伤在第二个检查 点之前不能完全修复, p53 转录激活的促调亡蛋白便 会清除不可修复细胞. 该研究还提示, 在低损伤强度 下 p53 促进细胞的存活, 为细胞凋亡设定了阈值. 该 工作揭示了一种高效的 p53 决定细胞命运的动力学 机制, 对进一步理解 p53 网络的工作机理具有重要 意义.

\section{5 p53 动力学模式与细胞命运的内在联系}

在 DNA 损伤响应过程中, p53 蛋白的动力学具 有多种模式. 探讨 $\mathrm{p} 53$ 动力学模式与细胞命运之间的 内在联系已成为近年来 $\mathrm{p} 53$ 动力学研究的热点. 哈佛 大学的 Lahav 研究组发现, 电离辐射可在 MCF-7 细 胞内引发一系列 p53 脉冲, 导致细胞周期阻断; 而紫 外辐射则在细胞内引起单一 $\mathrm{p} 53$ 脉冲或持续的 $\mathrm{p} 53$ 活化, 诱导细胞调亡 [43]. 他们还发现, 对细胞电离辐 射后, 通过多次施加 Mdm2 的抑制剂 Nutlin-3, 可将 p53 动力学由脉冲转变为与脉冲幅度相当的持续激 活. 相应地, 细胞命运由暂态的细胞周期阻断转变为 细胞衰老. 实验发现, $\mathrm{p} 53$ 动力学模式的改变可导致 下游靶基因表达的改变. p53 脉冲主要诱导与细胞周 期阻断和 DNA 修复相关基因的表达, 而持续的 p53 激活则能引起促衰老基因的表达. 他们提出, p53 动 力学模式可控制细胞命运. 然而, p53 动力学模式决 定细胞命运的分子机制还有待深入研究.

来自韩国和中国香港的研究者通过布尔网络建 模和能量面分析的方法, 确定对 p53 动力学有重要影 响的几个网络节点, 提出 p53 与 Mdm2, Wip1, Cyclin $\mathrm{G}$ 和 ATM 等的相互作用对调控 p53 动力学起了关键 作用 ${ }^{[44]}$. 基于理论分析, 他们推断在 MCF-7 细胞中, 加入 Nutlin-3 的同时, 通过 RNA 干扰抑制 Wip1 的 表达, 可将 $\mathrm{p} 53$ 振荡转变为持续的 $\mathrm{p} 53$ 激活, 并引起 细胞凋亡. 在随后的实验中, 研究者采用了三种不同 的实验手段, 即分别加入低浓度抗癌药物 etoposide、 加入 Nutlin-3 和对 Wip1 进行 RNA 干扰. 他们比较 了不同实验条件下的 $\mathrm{p} 53$ 动力学和细胞命运, 发现 Nutlin-3 与 Wip1 特异的 RNA 干扰协同作用时, p53
才能被持续激活, 并高效地杀死受治疗的细胞. 他们 提出, 反复出现的 $\mathrm{p} 53$ 脉冲可引起细胞周期阻断, 而 持续高水平的 p53 则会引起细胞死亡. 在 Lahav 等 人的研究中, 电离辐射结合 Nutlin-3 治疗导致细胞衰 老 ${ }^{[43]}$. 而该研究则发现, 进一步对 Wip1 进行 RNA 干 扰, 则导致细胞凋亡. 因此, 不同的 p53 动力学模式 可能对应于不同的 p53 激活程度; Nutlin-3 治疗结合 RNA 干扰带来的协同效应可能导致 p53 的完全激活, 进而触发细胞调亡.

\section{6 结论}

p53 作为重要的肿瘤抑制因子, 其信号网络对 DNA 损伤这类具有基因毒性的刺激的正确响应对维 护基因组的完整性具有极其重要的意义. 在响应过程 中, p53 针对不同类型和强度的刺激可能呈现不同的 动力学行为, 调控不同的细胞应激反应. p53 蛋白水 平、翻译后修饰状态及与辅助因子的相互作用等众 多的因素都会影响到 p53 的活性, 进而影响细胞的命 运 ${ }^{[7]}$. p53 动力学研究旨在揭示 $\mathrm{p} 53$ 如何将应激信号 转化为特定细胞命运的编码机制. 探讨作为输入信号 的细胞应激如何激发特定的 p53 动力学, 是研究 p 53 网络响应机制的第一步; 进一步则要研究 p53 动力学 是如何决定细胞命运的, 揭示 p53 动力学编码为细胞 命运的分子机制.

已有的研究表明, 在 DNA 轻度损伤的细胞中, p53 表现出脉冲动力学. 这一模式具有很好的灵活性, 既有利于细胞周期停滞期间损伤的有效修复, 又可避 免轻度损伤细胞的过度调亡 [45]. 在损伤极为严重的 细胞中, p53 水平可能在相对短的时间内上升到较高 水平, 通过表达促调亡基因或通过线粒体 p53 诱发调 亡 ${ }^{[44]}$. 在中等损伤强度的细胞中, p53 可能先经历数 个脉冲, 再跃迁到高水平稳态 ${ }^{[26]}$. 此外, 长时间持续 的 $\mathrm{p} 53$ 脉冲或中等水平 $\mathrm{p} 53$ 的持续激活可能引起细 胞衰老 ${ }^{[43]}$. $\mathrm{p} 53$ 的动力学具有细胞种类和刺激类型 特异性, 导致这一特异性的内在机制还有待进一步 研究. 揭示调亡诱发过程中转录依赖和非转录依赖 的 p53 活性的贡献, 也是值得深入研究的课题. 在癌 症的治疗过程中, 调控 p53 的动力学模式, 可能会有 效提高治疗效果. 


\section{参考文献}

1 Strogatz S. Nonlinear Dynamics and Chaos: With Applications to Physics, Biology, Chemistry and Engineering. Boulder: Westview, 2000

2 Boogerd F C, Bruggeman F J, Hofmeyr J H S, et al. Systems Biology: Philosophical Foundations. Amsterdam: Elsevier, 2007

3 Levine A J. p53, the cellular gatekeeper for growth and division. Cell, 1997, 88(3): 323-331

4 Vousden K H, Lu X. Live or let die: The cell's response to p53. Nat Rev Cancer, 2002, 2(8): 594-604

5 Vousden K H. Outcomes of p53 activation-spoilt for choice. J Cell Sci, 2006, 119(24): 5015-5020

6 Speidel D. Transcription-independent p53 apoptosis: An alternative route to death. Trends Cell Biol, 2009, 20(1): 14-24

7 Murray-Zmijewski F, Slee E A, Lu X. A complex barcode underlies the heterogeneous response of p53 to stress. Nat Rev Mol Cell Biol, 2008, 9(9): 702-712

8 Harris S L, Levine A J. The p53 pathway: Positive and negative feedback loops. Oncogene, 2005, 24(17): 2899-2908

9 Lev Bar-Or R, Maya R, Segel L A, et al. Generation of oscillations by the p53-Mdm2 feedback loop: A theoretical and experimental study. Proc Natl Acad Sci USA, 2000, 97(21): 11250

10 Lahav G, Rosenfeld N, Sigal A, et al. Dynamics of the p53-Mdm2 feedback loop in individual cells. Nat Genet, 2004, 36(2): 147-150

11 Vousden K H, Lane D P. p53 in health and disease. Nat Rev Mol Cell Biol, 2007, 8(4): 275-283

12 Batchelor E, Loewer A, Lahav G. The ups and downs of p53: Understanding protein dynamics in single cells. Nat Rev Cancer, 2009, 9(5): 371-377

13 Oda K, Arakawa H, Tanaka T, et al. p53AIP1, a potential mediator of p53-dependent apoptosis, and its regulation by Ser-46-phosphorylated p53. Cell, 2000, 102(6): 849-462

14 Okamura S, Arakawa H, Tanaka T, et al. p53DINP1, a p53-inducible gene, regulates p53-dependent apoptosis. Mol Cell, 2001, 8(1): 85-94

15 Mayo L D, Seo Y R, Jackson M W, et al. Phosphorylation of human p53 at serine 46 determines promoter selection and whether ppoptosis is attenuated or amplified. J Biol Chem, 2005, 280(28): 25953

16 Tomasini R, Samir A A, Carrier A, et al. TP53INP1s and homeodomain-interacting protein kinase-2 (HIPK2) are partners in regulating p53 activity. J Biol Chem, 2003, 278(39): 37722

17 Batchelor E, Loewer A, Mock C, et al. Stimulus-dependent dynamics of p53 in single cells. Mol Syst Biol, 2011, 7: 488

$18 \mathrm{Hu}$ W, Feng Z, Ma L, et al. A single nucleotide polymorphism in the Mdm2 gene disrupts the oscillation of p53 and Mdm2 levels in cells. Cancer Res, 2007, 67(6): 2757-2765

19 Ma L, Wagner J, Rice J J, et al. A plausible model for the digital response of p53 to DNA damage. Proc Natl Acad Sci USA, 2005, 102(40): 14266-14271

20 Batchelor E, Mock C S, Bhan I, et al. Recurrent initiation: A mechanism for triggering p53 pulses in response to DNA damage. Mol Cell, 2008, 30(3): 277-289

21 Ciliberto A, Novak B, Tyson J J. Steady states and oscillations in the p53/Mdm2 network. Cell Cycle, 2005, 4(3): 488-493

22 Zhang T, Brazhnik P, Tyson J J. Exploring mechanisms of the DNA-damage response - p53 pulses and their possible relevance to apoptosis. Cell Cycle, 2007, 6(1): 85-94

23 Loewer A, Batchelor E, Gaglia G, et al. Basal dynamics of p53 reveal transcriptionally attenuated pulses in cycling cells. Cell, 2010, 142(1): 89-100

24 Sun T, Yang W, Liu J, et al. Modeling the basal dynamics of p53 system. PLoS ONE, 2011, 6(11): e27882

25 Zhang X P, Liu F, Cheng Z, et al. Cell fate decision mediated by p53 pulses. Proc Natl Acad Sci USA, 2009, 106(30): 12245-12250

26 Zhang X P, Liu F, Wang W. Two-phase dynamics of p53 in the DNA damage response. Proc Natl Acad Sci USA, 2011, 108(22): 8990-8995

27 D’Orazi G, Cecchinelli B, Bruno T, et al. Homeodomain-interacting protein kinase-2 phosphorylates p53 at Ser 46 and mediates apoptosis. Nat Cell Biol, 2002, 4(1): 11-19

28 Rinaldo C, Prodosmo A, Mancini F, et al. MDM2-regulated degradation of HIPK2 prevents p53Ser46 phosphorylation and DNA damage-induced apoptosis. Mol Cell, 2007, 25(5): 739-750

29 Di Stefano V, Mattiussi M, Sacchi A, et al. HIPK2 inhibits both MDM2 gene and protein by, respectively, p53-dependent and independent regulations. FEBS Lett, 2005, 579(25): 5473-5480

30 Zhang X P, Liu F, Wang W. Interplay between Mdm2 and HIPK2 in the DNA damage response. J R Soc Interface, 2014, 11(96): 20140319

31 Xiao J, Lin H, Luo X, et al. miR-605 joins p53 network to form a p53:miR-605:Mdm2 positive feedback loop in response to stress. EMBO J, 
2011, 30(3): 524-532

32 Hermeking H. The miR-34 family in cancer and apoptosis. Cell Death Differ, 2010, 17(2): 193-199

33 Zhou C H, Zhang X P, Liu F, et al. Involvement of miR-605 and miR-34a in the DNA damage response promotes apoptosis induction. Biophys J, 2014, 106(8): 1792-1800

34 Das S, Raj L, Zhao B, et al. Hzf determines cell survival upon genotoxic stress by modulating p53 transactivation. Cell, 2007, 130(4): 624-637

35 Samuels-Lev Y, O’Connor D J, Bergamaschi D, et al. ASPP proteins specifically stimulate the apoptotic function of p53. Mol Cell, 2001, 8(4): 781-794

36 Zhang X P, Liu F, Wang W. Regulation of the DNA damage response by p53 cofactors. Biophys J, 2012, 102(10): 2251-2260

37 Polager S, Ginsberg D. p53 and E2f: Partners in life and death. Nat Rev Cancer, 2009, 9(10): 738-748

38 Zhang X P, Liu F, Wang W. Coordination between cell cycle progression and cell fate decision by the p53 and E2F1 pathways in response to DNA damage. J Biol Chem, 2010, 285(41): 31571-31580

39 Chipuk J E, Bouchier-Hayes L, Kuwana T, et al. PUMA couples the nuclear and cytoplasmic proapoptotic function of p53. Science, 2005, 309(5741): 1732-1735

40 Pu T, Zhang X P, Liu F, et al. Coordination of the nuclear and cytoplasmic activities of p53 in response to DNA damage. Biophys J, 2010, 99(6): 1696-1705

41 Erster S, Mihara M, Kim R H, et al. In vivo mitochondrial p53 translocation triggers a rapid first wave of cell death in response to DNA damage that can precede p53 target gene activation. Mol Cell Biol, 2004, 24(15): 6728-6741

42 Tian X J, Liu F, Zhang X P, et al. A two-step mechanism for cell fate decision by coordination of nuclear and mitochondrial p53 activities. PLoS One, 2012, 7(6): e38164

43 Purvis J E, Karhohs K W, Mock C, et al. p53 dynamics control cell fate. Science, 2012, 336(6087): 1440-1444

44 Choi M, Shi J, Jung S H, et al. Attractor landscape analysis reveals feedback loops in the p53 network that control the cellular response to DNA damage. Sci Signal, 2012, 5(251): ra83

45 Lahav G. The strength of indecisiveness: Oscillatory behavior for better cell fate determination. Sci STKE, 2004, 2004(264): pe55

\title{
Nonlinear dynamics in the p53 signaling network
}

\author{
ZHANG XiaoPeng ${ }^{1,2}$, LIU Feng $^{1 *} \&$ WANG Wei ${ }^{1 *}$ \\ ${ }^{1}$ School of Physics and National Laboratory of Solid State Microstructures, Nanjing 210093, China; \\ ${ }^{2}$ Kuang Yaming Honors School, Nanjing University, Nanjing 210093, China
}

The 553 protein is an important tumor suppressor that modulates many physiological activities of the cell and maintains the stability and integrity of the genome. p53 is located at the center of a complicated signaling network. The p53 network can respond to a wide variety of stress including DNA damage, oncogene activation and telomere erosion. Depending on the severity of DNA damage, p53 may induce DNA repair/cell cycle arrest, senescence, or apoptosis to avoid the replication and propagation of the genomic defects to daughter cells. The concentration of p53 exhibits several types of dynamics such as pulsatile and switch-like behaviors. There exist rich nonlinear properties in the p53 dynamics. In this review, we focus on the latest progress in the field of p53 dynamics, and clarify the underlying connection between p53 dynamics and its biological functions in the DNA damage response.

nonlinear dynamics, p53 pulses, apoptosis, cell cycle arrest, cell-fate decision

PACS: $87.16 . Y c, 05.45 .-\mathrm{a}, 87.16 . \mathrm{Xa}$

doi: $10.1360 /$ SSPMA2014-00121 